$577-586$

\title{
Long-term morbidity and mortality in patients diagnosed with an insulinoma
}

\author{
Elina Peltola $\mathbb{1}^{1,2}$, Päivi Hannula 1,3, Heini Huhtala4, Saara Metso1,3, Juhani Sand5, Johanna Laukkarinen 1,5, \\ Mirja Tiikkainen ${ }^{6}$, Jukka Sirén ${ }^{7,8}$, Minna Soinio ${ }^{9}$, Pirjo Nuutila ${ }^{10,11}$, Leena Moilanen ${ }^{12}$, David E Laaksonen ${ }^{12}$, \\ Tapani Ebeling ${ }^{13,14}$, Johanna Arola ${ }^{15,16}$, Camilla Schalin-Jäntti ${ }^{6,17}$ and Pia Jaatinen 1 1,2,3
}

${ }^{1}$ Faculty of Medicine and Health Technology, Tampere University, Tampere, Finland, ${ }^{2}$ Division of Internal Medicine, Seinäjoki Central Hospital, Seinäjoki, Finland, ${ }^{3}$ Endocrinology, Department of Internal Medicine, Tampere University Hospital, Tampere, Finland, ${ }^{4}$ Faculty of Social Sciences, Tampere University, Tampere, Finland, ${ }^{5}$ Department of Gastroenterology and Alimentary Tract Surgery, Tampere University Hospital, Tampere, Finland, ${ }^{6}$ Endocrinology, Abdominal Center, ${ }^{7}$ Surgery, Abdominal Center, Helsinki University Hospital, Helsinki, Finland, ${ }^{8}$ Surgery, Abdominal Center, University of Helsinki, Helsinki, Finland, ${ }^{9}$ Department of Endocrinology, ${ }^{10}$ Department of Endocrinology, Division of Medicine, Turku University Hospital, Turku, Finland, ${ }^{11}$ Turku PET Centre, University of Turku, Turku, Finland, ${ }^{12}$ Department of Medicine, Kuopio University Hospital, Kuopio, Finland, ${ }^{13}$ Faculty of Medicine, University of Oulu, Oulu, Finland, ${ }^{14}$ Endocrinology, Department of Medicine, Oulu University Hospital, Oulu, Finland, ${ }^{15}$ Pathology, HUSLAB, Helsinki University Hospital, Helsinki, Finland, ${ }^{16}$ Pathology, University of Helsinki, Helsinki, Finland, and ${ }^{17}$ Endocrinology, Abdominal Center, University of Helsinki, Helsinki, Finland

Correspondence should be addressed to E Peltola

Email elina.peltola@tuni.fi

\begin{abstract}
Objective: Insulinomas are rare functional pancreatic neuroendocrine tumours. As previous data on the long-term prognosis of insulinoma patients are scarce, we studied the morbidity and mortality in the Finnish insulinoma cohort. Design: Retrospective cohort study.

Methods: Incidence of endocrine, cardiovascular, gastrointestinal and psychiatric disorders, and cancers was compared in all the patients diagnosed with an insulinoma in Finland during 1980-2010 ( $n=79$, including two patients with multiple endocrine neoplasia type 1 syndrome), vs 316 matched controls, using the Mantel-Haenszel method. Overall survival was analysed with Kaplan-Meier and Cox regression analyses.

Results: The median length of follow-up was 10.7 years for the patients and 12.2 years for the controls. The long-term incidence of atrial fibrillation (rate ratio (RR): 2.07 (95\% Cl: 1.02-4.22)), intestinal obstruction (18.65 (2.09-166.86)), and possibly breast (4.46 (1.29-15.39) and kidney cancers (RR not applicable) was increased among insulinoma patients vs controls, $P<0.05$ for all comparisons. Endocrine disorders and pancreatic diseases were more frequent in the patients during the first year after insulinoma diagnosis, but not later on. The survival of patients with a nonmetastatic insulinoma $(n=70)$ was similar to that of controls, but for patients with distant metastases $(n=9)$, the survival was significantly impaired (median 3.4 years).

Conclusions: The long-term prognosis of patients with a non-metastatic insulinoma is similar to the general population, except for an increased incidence of atrial fibrillation, intestinal obstruction, and possibly breast and kidney cancers. These results need to be confirmed in future studies. Metastatic insulinomas entail a markedly decreased survival.
\end{abstract}

\section{Introduction}

Insulinomas are rare insulin-secreting functional pancreatic neuroendocrine tumours, with an estimated incidence of $1-4$ per million per year $(1,2,3,4)$. They usually do not show metastatic behaviour and are considered cured after complete surgical removal of the tumour $(3,4,5)$. On the other hand, disease recurrence occurs in $7 \%$ of the https://eje.bioscientifica.com https://doi.org/10.1530/EJE-21-0230 Printed in Great Britain

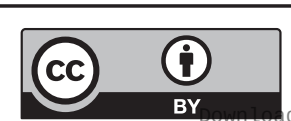

This work is licensed under a Creative Commons Attribution 4.0 International License. 
surgically treated patients (4), and in patients with distant metastases, the median survival has been reported to be less than 2 years (2).

Despitetheimproved diagnosticoptions, the diagnostic delay of insulinomas has remained long, presumably due to the rarity of the disease and the nonspecific clinical picture, as we have shown in our previous study on all adult patients diagnosed with an insulinoma in Finland during 1980-2010 (6). Because previous data on the long-term prognosis of insulinoma patients are scarce, we wanted to study the long-term morbidity and mortality in the Finnish insulinoma cohort (6).

\section{Subjects and methods}

The Finnish insulinoma register consists of all adult patients ( $\geq 18$ years of age) diagnosed with an insulinoma in Finland during 1980-2010 $(n=79)(6)$. For each patient, four controls were chosen from the Finnish Population Register Centre. The controls had to be equal by age $( \pm 6$ months), gender, and the place of residence, and alive at the diagnosis of the corresponding patient. The dates of death or emigration were provided by the Finnish Population Register Centre. Personal identification numbers assigned to all Finnish residents were used to link the information from the separate registers described below. The registerbased follow-up began on 1 January 1980 and lasted until 31 December 2015, unless death or emigration occurred first.

The morbidity of insulinoma patients vs controls was analysed before and after the diagnosis of insulinoma, focusing on five distinct disease groups: endocrine, gastrointestinal, cardiovascular, cancer, and psychiatric diseases, to evaluate the potential comorbidity and longterm effects of insulinoma on the development of these diseases. Cancer morbidity was evaluated based on the cancer diagnoses registered at the Finnish Cancer Registry, where Finnish health care organizations statutorily provide information on all new cancer cases. Insulinoma-related notifications were excluded from the analyses.

Morbidity due to endocrine, cardiovascular, gastrointestinal, and psychiatric disorders was evaluated on the basis of the diagnoses registered at the National Hospital Discharge Register, the Care Register for Health Care. This register, maintained by the Finnish Institute of Health and Welfare, collects the statutory data on all Finnish residents discharged from inpatient care in any Finnish hospital since 1969 and on outpatient visits in specialized health care since 1998. The diagnoses are coded according to the Finnish version of the 10th revision of the International Classification of Diseases (ICD-10) since 1996, ICD-9 during 1987-1995, and ICD-8 during 19801986. These classifications were reviewed, and the diagnoses of interest were grouped into corresponding disease categories and subcategories (Supplementary Table 1, see section on supplementary materials given at the end of this article). Both the primary and the secondary diagnoses were included in the analyses, and the diagnosis codes for hyperinsulinism and hypoglycaemia were excluded from the analysis of endocrine disorders.

For the mortality analyses, the causes of death were obtained from Statistics Finland, which collects the dates and causes of death of all Finnish citizens deceased since 1971. The causes of death are classified according to the ICD, as well as with a national time series classification, including 54 categories. In the analyses, we used the underlying cause of death, defined as the disease that has initiated the series of illnesses directly leading to death.

This study was conducted in accordance with the Declaration of Helsinki. The Regional Ethics Committee of the Tampere University Hospital catchment area reviewed and approved the study protocol. Informed consent was waived because of the retrospective, register-based nature of the study, and the fact that many of the study subjects died before data collection for the study. The Finnish Institute for Health and Welfare, the University Hospitals of Tampere, Helsinki, Kuopio, Oulu and Turku, Statistics Finland, and the Finnish Population Register Centre yielded permission for the use of data from their registers. Research data are not shared for ethical reasons, to protect the anonymity of patients with a rare disease.

\section{Statistical analysis}

The analyses were conducted with the IBM SPSS Statistics for Windows, Versions 25.0 and 27.0 (IBM Corp.), the STATA Statistical Software, Release 13 (StataCorp LP), and the OpenEPI Collection of Epidemiologic Calculators, Version 3.01. The data are presented as mean (S.D.) for normally distributed variables, median (minimum-maximum) for other numerical variables, and number (\%) for categorical variables.

In the morbidity analyses, the prevalence of diseases diagnosed before the diagnosis of insulinoma was first compared between the patients and the controls with the Fisher's exact test and conditional logistic regression. Then, the incidence rates of these disease groups after the diagnosis of insulinoma were compared by analysing the incidence rate ratios (RR) and 95\% CIs, using the MantelHaenszel method. Because only the first notification of each 
disease category per person was included in the analyses, the patients with a disease registered before the diagnosis of insulinoma were excluded from the incidence calculations of that disease category, together with their controls. The controls with a given disease diagnosed before the index date were excluded from the analyses individually. For diseases with a statistically significant difference in the patients vs controls, a sensitivity analysis was performed, excluding the MEN1 patients and their controls, as well as the persons with each disease diagnosed within the first year after insulinoma diagnosis, to eliminate detection bias. The Bonferroni correction was applied to define the level of significance for multiple comparisons.

The overall survival of the patients vs controls was compared using Kaplan-Meier analysis with the log-rank test. Insulinomas were retrospectively classified according to their highest diameter ( $\geq$ vs $<2 \mathrm{~cm}$ ) and staged according to the most recent TNM classification system (7). Cox regression analyses were used to calculate the hazard ratios (HR), to identify factors associated with mortality among the patients. The distribution of the causes of death was compared with the Fisher's exact test. For the patients who underwent curativeintent surgery, disease-free survival was calculated from the date of primary surgery to the date of disease progression or relapse. In the survival analyses, a two-sided $P$ value below 0.05 was considered statistically significant.

\section{Results}

Follow-up data of all the 79 patients and their 316 controls were included in the study. The mean age at the insulinoma diagnosis was 51.7 (15.6) years in the patients and 51.7 (15.5) years in the controls. The median duration of symptoms before the diagnosis was 13.0 (0.1-243.5) months. The median duration of the register-based follow-up between 1 January 1980, and the date of diagnosis of insulinoma was 22.7 (0.5-30.8) years for both the patients and the controls, and the median duration of follow-up after the diagnosis of insulinoma was $10.7(0.2-32.6)$ years for the patients and 12.2 (1.2-35.5) years for the controls. A metastatic insulinoma was detected in nine (11\%) patients. Multiple endocrine neoplasia type 1 (MEN1) syndrome was diagnosed in two patients, both associated with a non-metastatic, solitary insulinoma.

\section{Long-term morbidity}

Before the diagnosis of insulinoma, there was no statistically significant difference in morbidity between the patients and the controls (Supplementary Table 2). After the diagnosis of insulinoma, the overall incidence of any cardiovascular disease and theincidence of atrial fibrillation were increased in the patients vs controls, although the increase was not significant after the Bonferroni correction (Fig. 1A and Table 1). In the Kaplan-Meier analysis, the difference in the cumulative incidence of atrial fibrillation in the patients vs controls increased gradually after the diagnosis of insulinoma (Fig. 1B). A sensitivity analysis excluding the first year after insulinoma diagnosis showed a trend towards an increased incidence of atrial fibrillation in the insulinoma patients, but this difference was not statistically significant (Table 2).

Regarding endocrine morbidity (Fig. 1C), the overall incidence of endocrine and thyroid disorders was higher in the patients than in the controls (Fig. 1C and Table 1). The thyroid diagnoses included hypothyroidism in three (4\%), hyperthyroidism in two (3\%), and goitre in one (1\%) of the patients, compared to six (2\%), two (1\%), and two (1\%) of the controls, respectively. In the Kaplan-Meier analysis, the cumulative incidence of thyroid disorders in the patients vs controls started to increase right from the diagnosis of insulinoma (Fig. 1D). After excluding the

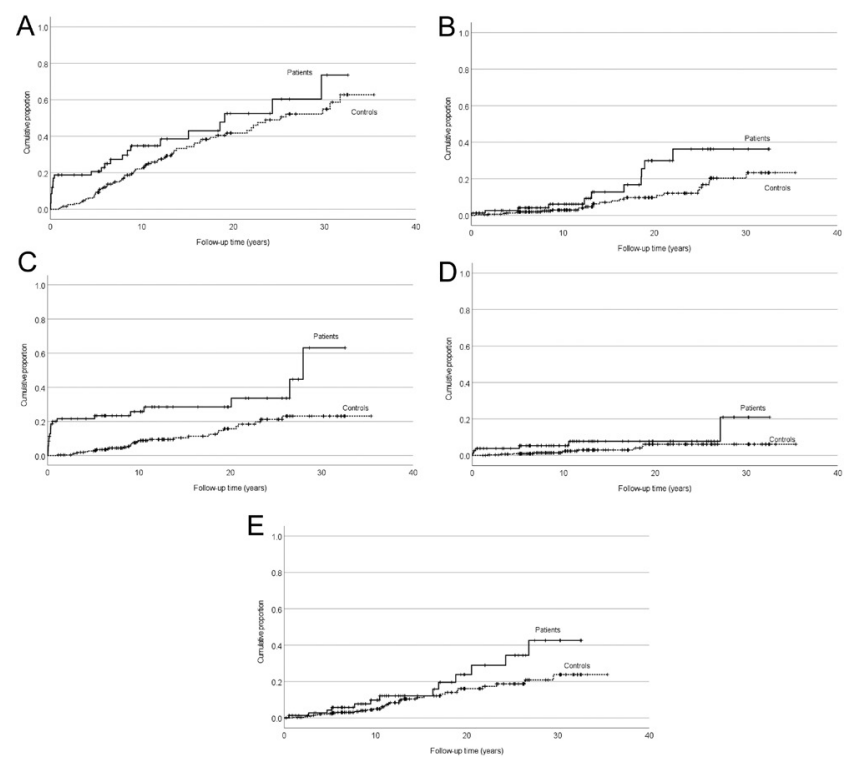

Figure 1

(A, B, C, D and E) Cumulative incidence of cardiovascular, endocrine, and cancer diseases in the patients (solid line) diagnosed with an insulinoma Finland during 1980-2010, compared with controls (dashed line) matched for age, gender, and the place of residence (log-rank test). ( $A$ ) Cardiovascular diseases $(P=0.048)$, (B) atrial fibrillation $(P=0.024)$, (C) endocrine disorders $(P<0.001)$, (D) thyroid disorders $(P=0.047)$, (E) cancers $(P=0.061)$. 


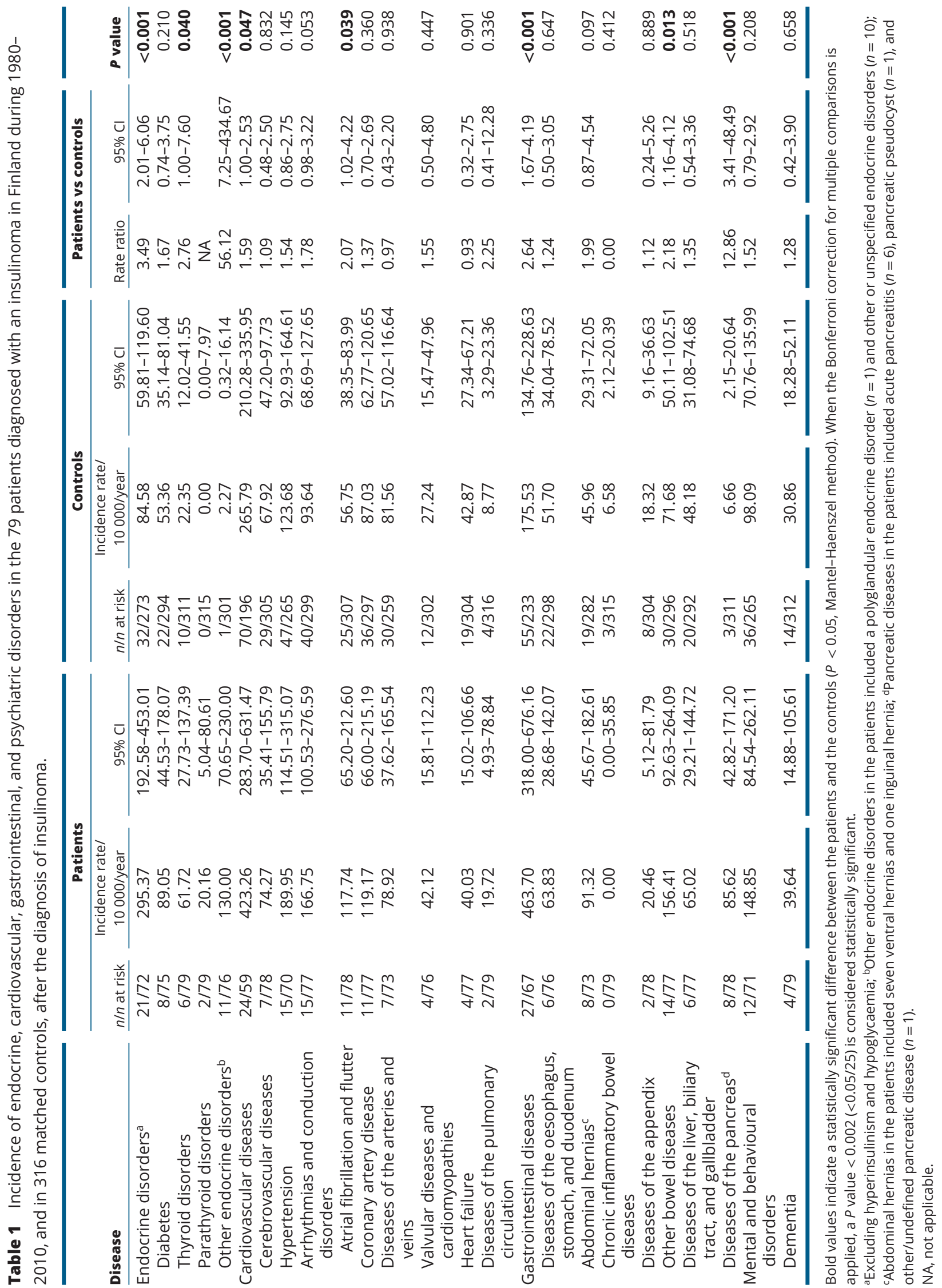




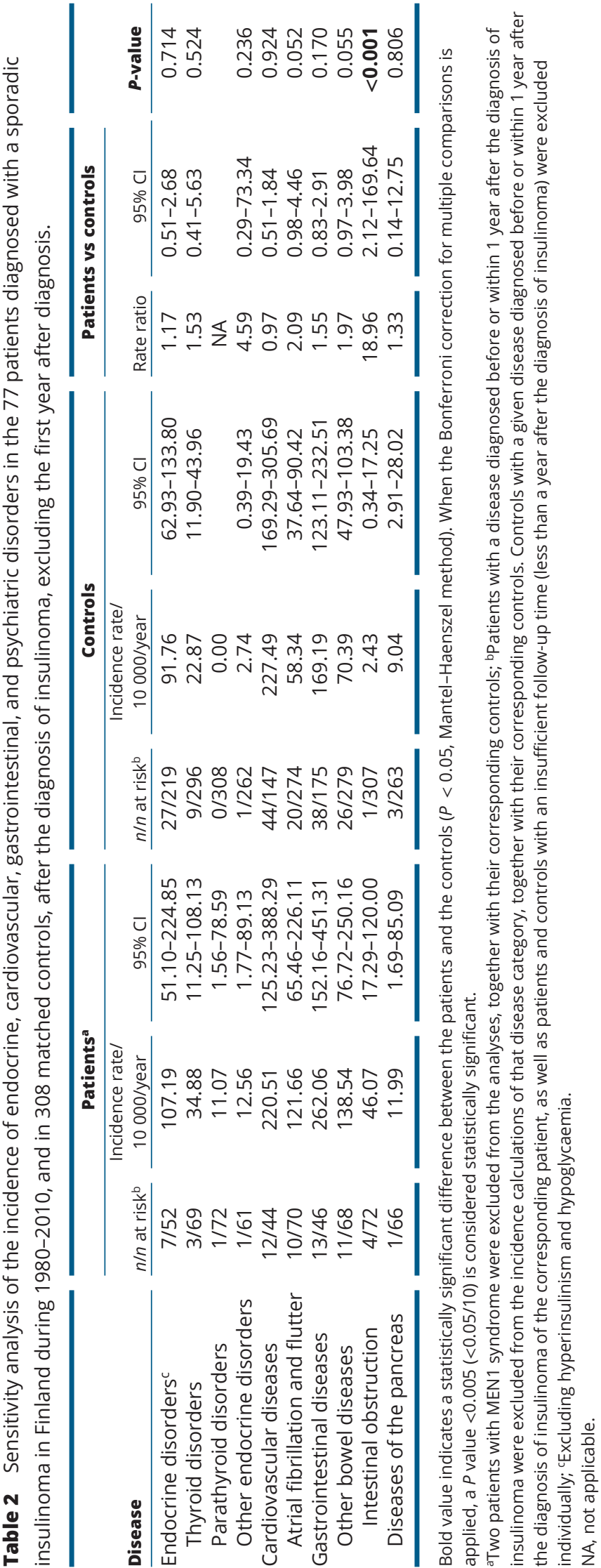

first post-diagnostic year, no difference was found in the incidence of endocrine or thyroid disorders between the patients and the controls (Table 2). A parathyroid disorder (hyperparathyroidism) was diagnosed in only two (3\%) patients, one of them having a confirmed MEN1 syndrome.

As for gastrointestinal diagnoses, the incidence of pancreatic diseases, and bowel diseases other than IBD, hernias, and appendiceal diseases, was increased among the insulinoma patients (Table 1). The increased incidence of pancreatic diseases was explained by acute pancreatitis, usually diagnosed during the first 2 months after the primary pancreatic surgery. In the subgroup analysis of bowel diseases, the only statistically significant difference was an increased incidence of intestinal obstruction, diagnosed in $5 \%$ of the surgically treated patients, a median of 5.9 (2.1-11.3) years after primary pancreatic surgery (RR: 18.7 (95\% CI: $2.1-166.9), P<0.001)$.

The incidence of dementia or all mental and behavioural disorders did not significantly differ between the patients and the controls (Table 1). Regarding cancer morbidity, 14 cancers were diagnosed in the patients and 42 in the controls (Fig. 1E and Table 3), after the diagnosis of insulinoma. Of specific cancer types, the incidence of breast and kidney cancers was increased in insulinoma patients vs controls (Table 3). After the exclusion of the 2 MEN1 patients and their controls, however, no statistically significant increase was found in breast cancer incidence (RR 2.64 (0.63-11.04), $P=0.167)$. The breast cancers occurred 4.7-24.3 years after the diagnosis of insulinoma. The three kidney cancers in the patients were diagnosed 2.7, 16.9, and 20.5 years after the diagnosis of a sporadic, non-metastatic insulinoma. Only one of the kidney cancers was detected before the end of insulinoma follow-up at the University Hospital.

\section{Long-term survival}

With the three disease progressions and three recurrences detected in the 71 patients treated with curative-intent surgery (6), the 5-, 10-, and 15-years disease-free survival rates were 94,93 , and $90 \%$, respectively. During the follow-up, 25 (32\%) patients and 63 (20\%) controls deceased. The Kaplan-Meier survival curves of the patients with a non-metastatic or a metastatic insulinoma and their controls are shown in Fig. 2. In a Cox regression analysis, the median overall survival of 27.5 (95\% CI: 24.1-30.8) years in the patients with a non-metastatic insulinoma did not significantly differ from the $33.2(29.8-36.7)$ years in their controls (HR: $1.5(0.9-2.6), P=0.128)$. In the patients with a metastatic insulinoma, the survival was significantly impaired, with a median of $3.4(2.9-4.0)$ years vs not 
reached in the controls (HR: $5.1(1.9-13.3), P=0.001)$. Three of the patients with metastatic insulinoma (33\%), however, showed a remarkably long survival time of 6-30 years.

In univariate analyses, older age, distant metastases, tumour size $\geq 2 \mathrm{~cm}$, higher preoperative serum insulin concentration, lack of curative-intent surgery, and the need for postoperative medication for the insulinoma were all associated with a significantly decreased overall survival among insulinoma patients (Supplementary Table 3). The occurrence of major surgical complications, classified as grades III-V of the Clavien-Dindo classification $(8,9)$, was associated with a decreased survival, due to the early postoperative mortality. After the exclusion of postoperative deaths (grade V complication), no significant difference was found in the survival of patients with major vs no or minor surgical complications (HR 2.28 (0.776.71), $P=0.136)$. The association of laparoscopic vs open surgery with survival could not be assessed as only two patients underwent laparoscopic insulinoma surgery. In the multivariate analyses, older age and distant metastases were associated with decreased survival (Table 4 and Supplementary Table 4).

\section{Causes of death}

Nine of the 25 insulinoma patients who deceased during the study period (36\%) died due to an insulinoma-related cause: 6 patients died of metastatic insulinoma, 2 patients died of surgical complications, and 1 patient died due to complications of invasive diagnostics, as previously described (6). With the two deaths due to surgical complications, the perioperative mortality, defined as any death occurring within 30 days after surgery, was $2.7 \%$. The causes of death of the patients and controls are presented in Table 5. During the follow-up, 16 non-insulinomarelated deaths occurred in the patients and 54 in the controls. Of these 16 deaths among the patients, 8 (50\%) were due to diseases of the circulatory system, 4 (25\%) due to cancer and 4 (25\%) due to other causes, compared to 24 (44\%), 12 (22\%) and 18 (33\%) in the controls, respectively $(P=0.765)$. The distribution of the causes of death did not significantly differ between the patients and the controls $(P=0.363)$, analysed according to the national time-series classification of Statistics Finland.

\section{Discussion}

This study suggests an increased long-term morbidity in insulinoma patients, due to atrial fibrillation, intestinal obstruction, and possibly breast and kidney cancers. 
Endocrine and pancreatic diseases were more frequent within the first year after the diagnosis, likely due to a detection bias and the occurrence of short-term surgical complications, respectively. Despite the increased longterm morbidity, the overall survival of patients with nonmetastatic insulinoma is similar to the general population. In patients with metastatic insulinoma, the prognosis is significantly impaired.

The long-term morbidity due to any cardiovascular disease, or due to atrial fibrillation was increased among the patients previously diagnosed with an insulinoma. The reason for the increased cardiovascular morbidity in insulinoma patients is unclear. Previous studies have found no association between insulinoma and hypertension $(10,11)$, but the incidence of atrial fibrillation in insulinoma patients has, to our knowledge, not been studied before. Hypoglycaemia has been shown to induce cardiac arrhythmias in persons with diabetes, but the potential effect of hypoglycaemia on the cardiovascular morbidity of insulinoma patients is unclear $(12,13)$. Unfortunately, the total burden of hyperinsulinaemia and hypoglycaemia could not be quantified retrospectively in the present study, nor in the previous ones. In our study, the follow-up due to insulinoma may have contributed to the early diagnoses of atrial fibrillation in the patients as the difference between the patients and the controls was no longer statistically significant after excluding the first year after diagnosis.

Morbidity due to any endocrine or thyroid disorders was increased among the patients during the first year after insulinoma diagnosis, but not later on. Although the diagnosis codes for hyperinsulinism and hypoglycaemia were excluded, the substantial number of other or unspecified endocrine disorders diagnosed near the time of diagnosis of insulinoma indicates that these codes may have been used instead of the specific diagnoses for insulinoma. A possible explanation for the increased thyroid morbidity is the careful examination and follow-up of these patients by endocrinologists, contributing to a prompt diagnosis of disorders that may partly remain undiagnosed in the general population.

The incidence of acute pancreatitis and intestinal obstruction was increased among the insulinoma patients vs controls. Most cases of pancreatitis occurred as early postoperative complications, with a rate of $10 \%$ in the surgically treated patients, as described previously (6). Intestinal obstruction occurred in $5 \%$ of the surgically treated patients, likely as a late postoperative complication of insulinoma surgery. Previous studies on surgically treated insulinomas have mainly focused on short-term complications and have not reported the incidence of late intestinal obstruction in insulinoma patients. In accordance with this study, previous studies have reported similar rates of intestinal obstruction after pancreatic and other abdominal surgery $(14,15,16)$.

Morbidity due to breast and kidney cancers also seemed to increase among insulinoma patients. To our knowledge, increased incidence of breast or kidney cancers in insulinoma patients has not been reported before, apart from an increased risk for breast cancer in MEN1 patients (17). The breast and kidney cancers of the patients were diagnosed 2.7-24.3 years after the diagnosis of insulinoma. The MEN1 syndrome explained at least part of the increased morbidity due to breast cancer, and no statistically significant difference was found in the breast cancer incidence of the patients vs controls after the exclusion of MEN1 patients from the analysis. As renal cancers are often discovered incidentally in abdominal imaging $(18,19)$, we cannot exclude neither a true association of kidney cancer with insulinoma nor an effect of frequent CT scanning. As these results were based on a small number of patients diagnosed with breast or kidney cancer, larger studies are needed to confirm these preliminary findings.

No significant difference was detected in the incidence of dementia or other psychiatric disorders between insulinoma patients and controls. The sample size and the follow-up time in our study may, however, have been insufficient to detect a possible difference. A recent prospective study reported cognitive impairment in 18 of 34 insulinoma patients, measured with the Montreal Cognitive Assessment questionnaire prior to the pancreatic surgery, with improvement detected in most patients at 1 year after surgery (20).
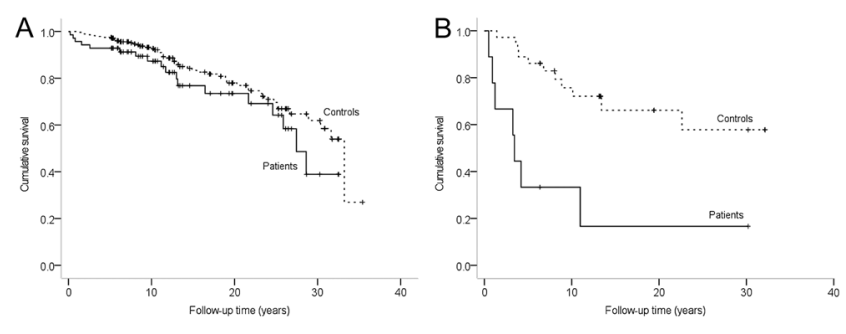

\section{Figure 2}

(A and B) Survival of the patients diagnosed with a nonmetastatic (A) or a metastatic insulinoma (B) in Finland during 1980-2010, compared with controls matched for age, gender, and the place of residence (log-rank test). (A) Patients with non-metastatic insulinoma vs controls ( $P=0.125)$, (B) patients with metastatic insulinoma vs controls $(P<0.001)$. 
Table 4 Multivariate analysis of factors associated with mortality among patients diagnosed with an insulinoma in Finland during $1980-2010\left(n=75^{a}\right)$.

\begin{tabular}{l}
\hline Variable \\
\hline Age at diagnosis \\
Tumour localization (head/neck vs body/tail) \\
Tumour size $(\geq 2 \mathrm{~cm}$ vs $<2 \mathrm{~cm})$ \\
Distant metastases
\end{tabular}

\begin{tabular}{c}
\hline Hazard ratio \\
\hline 1.05 \\
1.90 \\
2.49 \\
3.71 \\
\hline
\end{tabular}

\begin{tabular}{l}
\hline $\mathbf{9 5 \%} \mathbf{~ C l}$ \\
\hline $1.02-1.08$ \\
$0.72-5.03$ \\
$0.93-6.65$ \\
$1.18-11.67$ \\
\hline
\end{tabular}

\begin{tabular}{c}
\hline $\boldsymbol{P}$ value \\
\hline $\mathbf{0 . 0 0 3}$ \\
0.197 \\
0.070 \\
$\mathbf{0 . 0 2 5}$ \\
\hline
\end{tabular}

Bold values indicate a statistically significant hazard ratio $(P<0.05$, Cox proportional hazards model).

aFour of the 79 patients in the total cohort were excluded from this multivariate analysis, due to missing data regarding tumour size $(n=4)$ and localization $(n=2)$.

In the present study, the overall survival of patients with non-metastatic insulinoma did not significantly differ from the general population. The 10 -year survival of $87 \%$ for non-metastatic and $33 \%$ for metastatic insulinomas in this study was similar to the 91 and 29\%, respectively, reported previously (1). Similarly to previous studies, older age and metastases were the most important factors associated with an impaired survival $(1,21,22)$. In fact, recent evidence suggests that metastatic and non-metastatic insulinomas differ in their origin and pathogenesis and should be regarded as two different diseases (23). An increased risk of insulinoma recurrence and an impaired survival has been reported in MEN1 patients (1). In our study, however, no recurrences were detected during the follow-up of the two MEN1 patients.

Among the surgically treated insulinoma patients, the surgical method or the period of surgery was not associated with the overall survival. The postoperative mortality of $2.7 \%$ was slightly lower than the $3.7 \%$ reported previously for an open approach (4). Except for the postoperative deaths, no significant association was found between surgical complications and overall survival, which is in line with a recent series of 105 surgically managed pancreatic neuroendocrine tumour patients (24). A recent study of 198 insulinoma patients, however, reported a higher reoperation rate after tumour enucleations compared to pancreatic resections (25). To minimize the complication risks and need for reoperations, the invasive diagnostics and surgical treatment of insulinomas, like all pancreatic tumours, should be performed in centres with adequate expertise $(2,26,27,28,29,30,31)$.

In this study, the median overall survival of 3.4 years in patients with a metastatic insulinoma was similar to the 40 months (3.3 years) reported recently in 31 patients with metastatic insulinomas (32). This is better than the median survival of 29 months (2.4 years) reported earlier (22). Despite the poor overall survival, one-third of the patients with metastatic insulinoma had a remarkably long survival time. Previous studies have shown that palliative debulking surgery, and newer treatment options, such as peptide receptor radionuclide therapy and everolimus, may improve survival and relieve symptoms in patients with metastatic disease $(26,32,33$, $34,35,36)$. Because of the small number of metastatic insulinomas, we were not able to assess the effect of treatment on the survival of patients with metastatic insulinoma.

The major strengths of this study are the nationwide, unselected study cohort, including all the patients diagnosed with an insulinoma in Finland over a 3-decade period, and the long-term follow-up data of the patients and controls, matched for age, gender, and the place of residence. In Finland, it is mandatory to report the underlying causes of death to the Population Information System, and the hospital discharge diagnoses to the National Hospital Discharge Register, contributing to the complete, comprehensive, and high-quality data in these registers $(37,38)$.

Table 5 Causes of death of the patients diagnosed with an insulinoma in Finland during 1980-2010 and their control group matched for age, gender, and the place of residence. Data are presented as $n(\%)$.

\begin{tabular}{|c|c|c|}
\hline & Patients $(n=79)$ & Controls $(n=316)$ \\
\hline Deaths related to insulinoma & $9(11.4)$ & $0(0)$ \\
\hline Deaths due to metastatic insulinoma & $6(7.6)$ & $0(0)$ \\
\hline Deaths due to complications of the invasive diagnostics or pancreatic surgery & $3(3.8)$ & $0(0)$ \\
\hline Deaths due to diseases of the circulatory system & $8(10.1)$ & $29(9.2)$ \\
\hline Deaths due to tumours (other than insulinoma) & $4(5.1)$ & $13(4.1)$ \\
\hline Deaths due to other causes & $4(5.1)$ & $21(6.6)$ \\
\hline Alive at the end of follow-up & $54(68.4)$ & $253(80.1)$ \\
\hline
\end{tabular}


The relatively small sample size, due to the rarity of insulinomas, is the major limitation of this study. In addition, the prognosis of the subgroups of patients with metastatic, recurrent, or MEN1-related insulinomas could not be evaluated comprehensively. Due to the retrospective, register-based study design we were not able to specify the causative factors of the long-term morbidity in insulinoma patients. Another limitation is that the National Hospital Discharge Register only includes information on the hospital visits in the specialized health care system, which may lead to underestimation of the incidence of nonsevere diseases, treated mainly in the primary health care. On the other hand, detection bias probably contributed to the high incidence of non-severe endocrine disorders near the time of diagnosis of insulinoma in the patients.

In conclusion, the long-term prognosis of patients with a non-metastatic insulinoma seems to be similar to the general population, except for an increased incidence of atrial fibrillation, intestinal obstruction, and possibly breast and kidney cancers. Metastatic insulinomas are rare but generally entail a markedly decreased survival. To our knowledge, this is the first study to report findings of increased long-term morbidity in insulinoma patients. In the future, larger studies are needed to confirm these results.

\section{Supplementary materials}

This is linked to the online version of the paper at https://doi.org/10.1530/ EJE-21-0230.

\section{Declaration of interest}

The authors declare that there is no conflict of interest that could be perceived as prejudicing the impartiality of this study.

\section{Funding}

This work was supported by the Competitive State Research Financing of the Expert Responsibility Area of Tampere University Hospital (grant numbers 9U012, 5900/3225, 6000/3231; to E P, P J), the Medical Research Fund of Seinäjoki Central Hospital (grant numbers 1717/6043, 1717/6080; to E P, P J), the Seinäjoki City and Tampere University Research Funds (not numbered; to E P, P J), the Helsinki University Hospital Research Funds (grant number TYH2019254; to C S-J) and Finska Läkaresällskapet (not numbered; to C S-J). The funders had no role in study design, data collection or analysis, decision to publish, or preparation of the manuscript. Preliminary results of this study have been presented as an e-poster at the eECE2021 congress (39).

\section{Acknowledgements}

The authors thank Antti Piiroinen, Fia Sundelin, and Hanna Hämäläinen for collecting the insulinoma register data from the patient records in Turku, Oulu, and Kuopio University Hospital catchment areas, respectively, and Esko Väyrynen for revising the language of the manuscript.

\section{References}

1 Service FJ, McMahon MM, O’Brien PC \& Ballard DJ. Functioning insulinoma: incidence, recurrence, and long-term survival of patients: a 60-year study. Mayo Clinic Proceedings 199166 711-719. (https://doi. org/10.1016/s0025-6196(12)62083-7)

2 Jensen RT, Cadiot G, Brandi ML, de Herder WW, Kaltsas G, Komminoth P, Scoazec JY, Salazar R, Sauvanet A, Kianmanesh R et al. ENETS consensus guidelines for the management of patients with digestive neuroendocrine neoplasms: functional pancreatic endocrine tumor syndromes. Neuroendocrinology 201295 98-119. (https://doi.org/10.1159/000335591)

3 Maggio I, Mollica V, Brighi N, Lamberti G, Manuzzi L, Ricci AD \& Campana D. The functioning side of the pancreas: a review on insulinomas. Journal of Endocrinological Investigation 202043 139-148. (https://doi.org/10.1007/s40618-019-01091-w)

4 Mehrabi A, Fischer L, Hafezi M, Dirlewanger A, Grenacher L, Diener MK, Fonouni H, Golriz M, Garoussi C, Fard N et al. A systematic review of localization, surgical treatment options, and outcome of insulinoma. Pancreas 201443 675-686. (https://doi.org/10.1097/ MPA.0000000000000110)

5 Falconi M, Eriksson B, Kaltsas G, Bartsch DK, Capdevila J, Caplin M, Kos-Kudla B, Kwekkeboom D, Rindi G, Klöppel G et al. ENETS consensus guidelines update for the management of patients with functional pancreatic neuroendocrine tumors and non-functional pancreatic neuroendocrine tumors. Neuroendocrinology 2016103 153-171. (https://doi.org/10.1159/000443171)

6 Peltola E, Hannula P, Huhtala H, Metso S, Kiviniemi U, Vornanen M, Sand J, Laukkarinen J, Tiikkainen M, Schalin-Jäntti C et al. Characteristics and outcomes of 79 patients with an insulinoma: a nationwide retrospective study in Finland. International Journal of Endocrinology 20182018 2059481. (https://doi. org/10.1155/2018/2059481)

7 Brierley JD, Gospodarowicz MK, Wittekind C, O'Sullivan B, Mason M, Asamura H, Lee A, Van Eycken E, Denny L, Amin MB et al. TNM Classification of Malignant Tumours, 8th ed., pp. 102-103. Oxford, UK; Hoboken, NJ: John Wiley \& Sons, Inc., 2017.

8 Clavien PA, Barkun J, de Oliveira ML, Vauthey JN, Dindo D, Schulick RD, de Santibanes E, Pekolj J, Slankamenac K, Bassi C et al. The Clavien-Dindo classification of surgical complications: fiveyear experience. Annals of Surgery 2009250 187-196. (https://doi. org/10.1097/SLA.0b013e3181b13ca2)

9 Dindo D, Demartines N \& Clavien PA. Classification of surgical complications: a new proposal with evaluation in a cohort of 6336 patients and results of a survey. Annals of Surgery 2004240 205-213. (https://doi.org/10.1097/01.sla.0000133083.54934.ae)

10 O’Brien T, Young Jr WF, Palumbo PJ, O'Brien PC \& Service FJ. Hypertension and dyslipidemia in patients with insulinoma. Mayo Clinic Proceedings 199368 141-146. (https://doi.org/10.1016/s00256196(12)60161-x)

11 Leonetti F, Iozzo P, Giaccari A, Sbraccia P, Buongiorno A, Tamburrano G \& Andreani D. Absence of clinically overt atherosclerotic vascular disease and adverse changes in cardiovascular risk factors in 70 patients with insulinoma. Journal of Endocrinological Investigation 199316 875-880. (https://doi. org/10.1007/BF03348949)

12 The International Hypoglycaemia Study Group. Hypoglycaemia, cardiovascular disease, and mortality in diabetes: epidemiology, pathogenesis, and management. Lancet: Diabetes and Endocrinology 20197 385-396. (https://doi.org/10.1016/S2213-8587(18)30315-2)

13 Ko SH, Park YM, Yun JS, Cha SA, Choi EK, Han K, Han E, Lee YH \& Ahn YB. Severe hypoglycemia is a risk factor for atrial fibrillation in type 2 diabetes mellitus: nationwide population-based cohort study. Journal of Diabetes and Its Complications 201832 157-163. (https://doi. org/10.1016/j.jdiacomp.2017.09.009)

14 ten Broek RPG, Issa Y, van Santbrink EJP, Bouvy ND, Kruitwagen RFPM, Jeekel J, Bakkum EA, Rovers MM \& van Goor H. Burden of adhesions 
in abdominal and pelvic surgery: systematic review and met-analysis. BMJ 2013347 f5588. (https://doi.org/10.1136/bmj.f5588)

15 Brown JA, Zenati MS, Simmons RL, Al Abbas AI, Chopra A, Smith K, Lee KKW, Hogg ME, Zeh HJ, Paniccia A et al. Long-term surgical complications after pancreatoduodenectomy: incidence, outcomes, and risk factors. Journal of Gastrointestinal Surgery 202024 1581-1589. (https://doi.org/10.1007/s11605-020-04641-3)

16 Norrbom C, Steding-Jessen M, Agger CT, Osler M, Krabbe-Sorensen M, Settnes A, Nilas L \& Loekkegaard ECL. Risk of adhesive bowel obstruction after abdominal surgery: a national cohort study of 665,423 Danish women. American Journal of Surgery 2019217 694-703. (https://doi.org/10.1016/j.amjsurg.2018.10.035)

17 van Leeuwaarde RS, Dreijerink KM, Ausems MG, Beijers HJ, Dekkers OM, de Herder WW, van der Horst-Schrivers AN, Drent ML, Bisschop PH, Havekes B et al. MEN1-dependent breast cancer: indication for early screening? Results from the Dutch MEN1 Study Group. Journal of Clinical Endocrinology and Metabolism 2017102 2083-2090. (https://doi.org/10.1210/jc.2016-3690)

18 Barrett TW, Schierling M, Zhou C, Colfax JD, Russ S, Conatser P, Lancaster P \& Wrenn K. Prevalence of incidental findings in trauma patients detected by computed tomography imaging. American Journal of Emergency Medicine 200927 428-435. (https://doi.org/10.1016/j. ajem.2008.03.025)

19 Znaor A, Lortet-Tieulent J, Laversanne M, Jemal A \& Bray F. International variations and trends in renal cell carcinoma incidence and mortality. European Urology 201567 519-530. (https://doi. org/10.1016/j.eururo.2014.10.002)

20 Dai H, Chen H, Hong X, Han X, Xu Q, Pang H, Yuan J, Wang X, Xu P, Jiang J et al. Early detection of cognitive impairment in patients with insulinoma. Endocrine 201965 524-530. (https://doi.org/10.1007/ s12020-019-01994-x)

21 Nikfarjam M, Warshaw AL, Axelrod L, Deshpande V, Thayer SP, Ferrone CR \& Fernandez-del Castillo C. Improved contemporary surgical management of insulinomas: a 25-year experience at the Massachusetts General Hospital. Annals of Surgery 2008247 165-172. (https://doi.org/10.1097/SLA.0b013e31815792ed)

22 Keutgen XM, Nilubol N \& Kebebew E. Malignant-functioning neuroendocrine tumors of the pancreas: a survival analysis. Surgery 2016159 1382-1389. (https://doi.org/10.1016/j.surg.2015.11.010)

23 Hackeng WM, Schelhaas W, Morsink FHM, Heidsma CM, van Eeden S, Valk GD, Vriens MR, Heaphy CM, Nieveen van Dijkum EJM, Offerhaus GJA et al. Alternative lengthening of telomeres and differential expression of endocrine transcription factors distinguish metastatic and non-metastatic insulinomas. Endocrine Pathology 2020 31 108-118. (https://doi.org/10.1007/s12022-020-09611-8)

24 Valente R, Lykoudis P, Tamburrino D, Inama M, Passas I, Toumpanakis C, Luong TV, Davidson B, Imber C, Malagò M et al. Major postoperative complications after pancreatic resection for P-NETS are not associated to earlier recurrence. European Journal of Surgical Oncology 201743 2119-2128. (https://doi.org/10.1016/j.ejso.2017.07.012)

25 Crippa S, Zerbi A, Boninsegna L, Capitanio V, Partelli S, Balzano G, Pederzoli P, Di Carlo V \& Falconi M. Surgical management of insulinomas: short- and long-term outcomes after enucleations and pancreatic resections. Archives of Surgery 2012147 261-266. (https:// doi.org/10.1001/archsurg.2011.1843)

26 Partelli S, Bartsch DK, Capdevila J, Chen J, Knigge U, Niederle B, Nieveen van Dijkum EJM, Pape UF, Pascher A, Ramage J et al. ENETS consensus guidelines for standard of care in neuroendocrine tumours: surgery for small intestinal and pancreatic neuroendocrine tumours. Neuroendocrinology 2017105 255-265. (https://doi. org/10.1159/000464292)

27 Ahola R, Sand J \& Laukkarinen J. Centralization of pancreatic surgery improves results: review. Scandinavian Journal of Surgery 2020109 4-10. (https://doi.org/10.1177/1457496919900411)

28 Ahola R, Sand J \& Laukkarinen J. Pancreatic resections are not only safest but also most cost-effective when performed in a high-volume centre: a Finnish Register Study. Pancreatology 201919 769-774. (https://doi.org/10.1016/j.pan.2019.06.007)

29 Antila A, Ahola R, Sand J \& Laukkarinen J. Management of postoperative complications may favour the centralization of distal pancreatectomies: nationwide data on pancreatic distal resections in Finland 2012-2014. Pancreatology 201919 26-30. (https://doi. org/10.1016/j.pan.2018.11.012)

30 Ahola R, Siiki A, Vasama K, Vornanen M, Sand J \& Laukkarinen J. Effect of centralization on long-term survival after resection of pancreatic ductal adenocarcinoma. British Journal of Surgery 2017 104 1532-1538. (https://doi.org/10.1002/bjs.10560)

31 de Wilde RF, Besselink MGH, van der Tweel I, de Hingh IHJT, van Eijck CHJ, Dejong CHC, Porte RJ, Gouma DJ, Busch ORC $\&$ Molenaar IQ. Impact of nationwide centralization of pancreaticoduodenectomy on hospital mortality. British Journal of Surgery 201299 404-410. (https://doi.org/10.1002/bjs.8664)

32 Veltroni A, Cosaro E, Spada F, Fazio N, Faggiano A, Colao A, Pusceddu S, Zatelli MC, Campana D, Piovesan A et al. Clinicopathological features, treatments and survival of malignant insulinomas: a multicenter study. European Journal of Endocrinology 2020182 439-446. (https://doi.org/10.1530/EJE-19-0989)

33 Tiensuu Janson E, Sorbye H, Welin S, Federspiel B, Gronbaek H, Hellman P, Ladekarl M, Langer SW, Mortensen J, SchalinJäntti $C$ et al. Nordic guidelines for diagnosis and treatment of gastroenteropancreatic neuroendocrine neoplasms. Acta Oncologica 201453 1284-1297. (https://doi.org/10.3109/0284186X.2014.941999)

34 Sada A, Glasgow AE, Vella A, Thompson GB, McKenzie TJ \& Habermann EB. Malignant insulinoma: a rare form of neuroendocrine tumor. World Journal of Surgery 202044 2288-2294. (https://doi. org/10.1007/s00268-020-05445-x)

35 Bernard V, Lombard-Bohas C, Taquet MC, Caroli-Bosc FX, Ruszniewski P, Niccoli P, Guimbaud R, Chougnet CN, Goichot B, Rohmer V, French Group of Endocrine Tumors et al. Efficacy of everolimus in patients with metastatic insulinoma and refractory hypoglycemia. European Journal of Endocrinology 2013168 665-674. (https://doi.org/10.1530/EJE-12-1101)

36 Brown E, Watkin D, Evans J, Yip V \& Cuthbertson DJ. Multidisciplinary management of refractory insulinomas. Clinical Endocrinology 201888 615-624. (https://doi.org/10.1111/cen.13528)

37 Official Statistics of Finland. Quality Descriptions, Deaths 2019. Helsinki: Statistics Finland, 2020. (available at: http://www.stat.fi/til/ kuol/2019/kuol_2019_2020-04-24_laa_001_en.html). Accessed on 23 September 2020.

38 Sund R. Quality of the Finnish hospital discharge register: a systematic review. Scandinavian Journal of Public Health 201240 505-515. (https:// doi.org/10.1177/1403494812456637)

39 Peltola E, Hannula P, Huhtala H, Metso S, Sand J, Laukkarinen J, Tiikkainen M, Sirén J, Soinio M, Nuutila P et al. Long-term prognosis in patients with insulinoma. Endocrine Abstracts 202173 PEP9.1. (https://doi.org/10.1530/endoabs.73.PEP9.1)

Received 1 March 2021

Revised version received 9 July 2021

Accepted 6 August 2021 